The Astrophysical Journal, 194:313-322, 1974 December 1

(c) 1974. The American Astronomical Society. All rights reserved. Printed in U.S.A.

\title{
DIFFUSE INTERSTELLAR BAND FORMATION IN DENSE CLOUDS
}

\author{
TheOdore P. SNOW, JR.*† \\ Princeton University Observatory \\ AND \\ JUDITH G. COHEN \\ Kitt Peak National Observatorył \\ Received 1974 May 20
}

\begin{abstract}
Data are presented which show that the strengths of the diffuse interstellar bands at 4430, 5780, and $5797 \AA$ tend to be deficient with respect to color excess in the lines of sight to stars lying behind dense interstellar clouds. In addition, diffuse-band data for 10 stars in the well-studied $\rho$ Ophiuchi cloud complex are presented, and systematic variations of band strengths with optical depth are discussed. It is found that the band strength per grain in the line of sight apparently decreases with increasing grain size, taking the ratio of total-to-selective absorption and the wavelength of maximum polarization as indicators of the latter. It is concluded that diffuseband formation probably takes place most efficiently in the outer regions of interstellar clouds. This may be consistent with a diffuse-band origin in small grains which are coated by mantles in cloud interiors; or in some molecule or molecules which are most abundant in the outer portions of interstellar clouds.

Subject headings: interstellar extinction - interstellar matter
\end{abstract}

\section{INTRODUCTION}

In the many years which have passed since the interstellar diffuse bands were first recognized (Merrill 1934, 1936; Merrill and Wilson 1938), observational studies have yielded very few unambiguous characteristics of the bands which may be used to identify their origin. Many of these observational studies have included examinations of correlations between band strengths and other interstellar quantities such as reddening (e.g., Duke 1951) and polarization (e.g., Krzemiński 1959). The apparent general correlation with interstellar extinction has led to a widespread hypothesis that the bands are formed in solid grains. There is no lack of experimental and theoretical arguments favoring the physical consistency of such a hypothesis, but the combination of ambiguity in the predictions of the models and imprecision in the measurements of the diffuse bands has resulted in a failure to find convincing evidence for any specific source over the others.

It has been suggested by Wampler (1966) that the broad band centered at $4430 \AA$ tends to be weakened with respect to $E_{B V}$ color excess in the spectra of stars situated behind dark clouds. This could have important implications for models of band formation which attribute the features to absorption by solid grains, because the nature of the grains in dense clouds may

* Visiting Astronomer, Kitt Peak National Observatory, which is operated by the Associated Universities for Research in Astronomy, Inc., under contract with the National Science Foundation.

$\dagger$ Part of this study was carried out by the author at the University of Washington.

$\ddagger$ Operated by the Associated Universities for Research in Astronomy, Inc., under contract with the National Science Foundation. be modified by the accretion of mantles (Field, Goldsmith, and Habing 1969; Mészáros 1972; Cohen 1973; Wallerstein and Goldsmith 1974; and Field 1974). We have undertaken the present study in hopes of examining in more detail the behavior of the diffuse bands in dark clouds. We have not only compared the correlations of band strengths with color excess for lines of sight in and out of dark clouds, but we have also determined how the band strengths vary with optical depth and probable grain size within a single cloud complex.

\section{THE DATA}

\section{a) Star Selections and Observations}

Our primary list of stars known to be lying behind dark clouds is taken from Cohen (1973). Briefly, the criteria used in determining whether a star belonged on her list of dark-cloud stars were that it lie near the center of one of the clouds in the catalog of Lynds (1962), that it be an O or B star with $E_{B V}$ color excess greater than $0.5 \mathrm{mag}$, and that it be brighter than $m_{V}=9.0 \mathrm{mag}$. Five stars in the IC 348 region of the Perseus II complex were added to the dark-cloud star list for this study. These five stars are fainter than 9th magnitude, and are $\mathrm{A}$ and early $\mathrm{F}$ stars.

In addition to the dark-cloud list, several stars known to lie behind less optically thick portions of large cloud complexes were observed. These include a few stars in the Perseus II region and several in the $\rho$ Ophiuchi cloud complex. The $\rho \mathrm{Oph}$ cloud is of particular interest because information is available on the variation of extinction and polarization properties within the cloud (Carrasco, Strom, and Strom 1973; referred to hereafter as CSS). We include here diffuse-band data on 10 stars in the $\rho$ Oph cloud which are described by CSS. 
TABLE 1

Dark Cloud Stars

\begin{tabular}{|c|c|c|c|c|c|c|c|}
\hline Star & Sp. Type & $V$ & $E_{B V}$ & $A_{c}(\lambda 4430)$ & $E W(\lambda 5780)$ & $E W(\lambda 5797)$ & Comments \\
\hline HD 21483. & B3 III & 7.06 & 0.58 & 6.0 & 0.28 & 0.16 & $\ldots$ \\
\hline $\mathrm{BD}+31^{\circ} 643$. & B5 V & 8.5 & 0.93 & 7.5 & 0.28 & 0.15 & $\ldots$ \\
\hline LF $24^{*} \ldots \ldots \ldots \ldots$ & B3 & 9.14 & 0.93 & 8 & $\ldots$ & $\ldots$ & $\ldots$ \\
\hline $\mathrm{G} 6 \dagger \ldots \ldots \ldots \ldots \ldots$ & F0 III-IV & 11.92 & 0.52 & $<3$ & $\ldots$ & $\ldots$ & $\ldots$ \\
\hline $\mathrm{G} 12 \uparrow \ldots \ldots \ldots \ldots$ & A2 III & 10.20 & 0.81 & 7 & $\ldots$ & $\ldots$ & $\ldots$ \\
\hline G16 $\ldots \ldots \ldots \ldots \ldots$ & A0 & 11.22 & 1.05 & 8 & $\ldots$ & $\ldots$ & $\ldots$ \\
\hline $\mathrm{G} 11 \uparrow \ldots \ldots \ldots \ldots$ & F0m: & 10.62 & 0.64 & 5 & $\ldots$ & $\ldots$ & $\therefore$ \\
\hline HD $46883 \ldots \ldots \ldots$ & B2 V & 7.9 & 0.52 & 12.0 & & & \\
\hline HD $147701 \ldots \ldots \ldots$ & B5 V & 8.35 & 0.72 & 7.0 & 0.38 & 0.11 & $\rho$ Oph Cloud \\
\hline HD $147888 \ldots \ldots \ldots$ & B3 V & 6.74 & 0.53 & 5.0 & 0.23 & 0.08 & $\rho$ Oph Cloud \\
\hline HD $147889 \ldots \ldots \ldots$ & B2 V & 7.86 & 1.08 & 10.0 & 0.45 & 0.22 & $\rho$ Oph Cloud \\
\hline HD $172028 \ldots \ldots \ldots$ & B3 II & 7.83 & 0.76 & 4.0 & & & $\ldots$ \\
\hline HD $203938 \ldots \ldots \ldots$ & B0.5 IV & 7.08 & 0.71 & 11.0 & 0.39 & 0.12 & ... \\
\hline
\end{tabular}

* IC 348 star (see Fredrick 1956). The $\lambda 4430$ data was provided by Strom.

† IC 348 star (see Harris, Morgan, and Rowan 1954). The $\lambda 4430$ data was provided by S. E. Strom.

Two of the stars in Cohen's (1973) "original list" of dark-cloud stars have been transferred in this study to the list of stars lying behind less optically thick portions of cloud complexes, because both stars have $E_{B V}$ color excesses slightly less than $0.5 \mathrm{mag}$.

The observations for most stars in the present study have been described elsewhere (Cohen 1973; Snow 1973). The $\lambda 4430$ data on the five stars in the IC 348 region were provided by Strom (1974), who obtained IIa-O image-tube spectra with the Cassegrain spectrograph on the 214-cm telescope at Kitt Peak National Observatory.

\section{b) Reduction and Errors}

Tracings were prepared of all the spectra, and the strengths of the features at 4430, 5780, and $5797 \AA$ were measured. Comparison of our data on $\lambda 4430$ with those of other observers for stars measured in common shows that the standard error in the central depth measurements is roughly \pm 1.5 percent of the continuum level. For $\lambda \lambda 5780$ and 5797 the error is estimated to be \pm 15 percent of the true equivalent width for features with equivalent widths greater than $40 \mathrm{~m} \AA$, and $\pm 10 \mathrm{~m} \AA$ for weaker features.
Our data on "dark cloud" stars and "other cloud" stars are presented in tables 1 and 2 , respectively. For some of the stars in table 2 , we have augmented the diffuse-band data with published results of other authors. In the cases where we have used $\lambda 4430$ strengths measured by others, we have allowed for observational bias by converting all measurements to a common system through linear transformations such as those derived by Walker (1963), Deeming and Walker (1967), and Snow (1973). For $\lambda \lambda 5780$ and 5797, where systematic bias among different observers is not apparent, data from other authors were used in this study without correction.

\section{c) Baseline Correlations}

In order to establish a baseline against which to compare the diffuse band strengths in dark clouds, we have used data on distant supergiants to establish a "normal" correlation between the band strengths and color excess. The stars used for this purpose were taken from Snow (1973) for the $4430 \AA$ band, and from Merrill et al. (1937) and Wu (1972) for $\lambda 5780$. The $\lambda 4430$ data on distant supergiants were augmented whenever possible by measurements of the same stars

TABLE 2

Other Cloud STARS

\begin{tabular}{|c|c|c|c|c|c|c|c|}
\hline Name & HD & Sp. Type & $E_{B V}$ & $A_{c}(\lambda 4430)$ & $E W(\lambda 5780)$ & $E W(\lambda 5797)$ & Comments \\
\hline$\ldots \ldots \ldots \ldots$ & 21856 & B1 V & 0.23 & 4.4 & $\ldots$ & $\ldots$ & \\
\hline$\ldots \ldots \ldots \ldots$ & 22951 & B0.5 V & 0.29 & 2.3 & & $\ldots$ & $\ldots$ \\
\hline$\ldots \ldots \ldots \ldots$ & 23060 & B2 Vp & 0.34 & 5.4 & 0.13 & & $\ldots$ \\
\hline o Per............. & 23180 & B1 III & 0.32 & 2.8 & 0.07 & 0.00 & $\cdots$ \\
\hline$\ldots \quad \ldots \ldots \ldots \ldots$ & 24131 & B1 V & 0.25 & 4.1 & & & \\
\hline$\zeta$ Per.......... & 24398 & B1 Ib & 0.38 & 3.4 & 0.20 & 0.11 & . \\
\hline$\xi$ Per........... & 29412 & O7 & 0.33 & 5.6 & 0.22 & 0.10 & .. \\
\hline$\delta \mathrm{Sco}$. & 143275 & B0 V & 0.17 & 4.5 & 0.28 & 0.13 & $\rho$ Oph Cloud \\
\hline$\beta^{1}$ Sco.......... & 144217 & B0.5 V & 0.23 & 4.7 & 0.25 & 0.11 & $\rho$ Oph Cloud \\
\hline$\omega^{1}$ Sco.......... & 144470 & B1 V & 0.22 & 4.4 & 0.20 & & $\rho$ Oph Cloud \\
\hline$\nu^{1}$ Sco........... & 145502 & B2 IV & 0.28 & 3.7 & 0.27 & 0.14 & $\rho$ Oph Cloud \\
\hline$\sigma \mathrm{Sco} \ldots \ldots \ldots \ldots$ & 147165 & B1 III & 0.39 & 5.6 & 0.38 & 0.12 & $\rho$ Oph Cloud \\
\hline$\rho$ Oph C $\ldots \ldots \ldots$ & 147932 & B5 V & 0.48 & 5.5 & & & $\rho$ Oph Cloud \\
\hline$\rho$ Oph A/B $\ldots \ldots$ & $147933 / 4$ & B2 IV/V & 0.48 & 8.2 & 0.28 & 0.07 & $\rho$ Oph Cloud \\
\hline
\end{tabular}



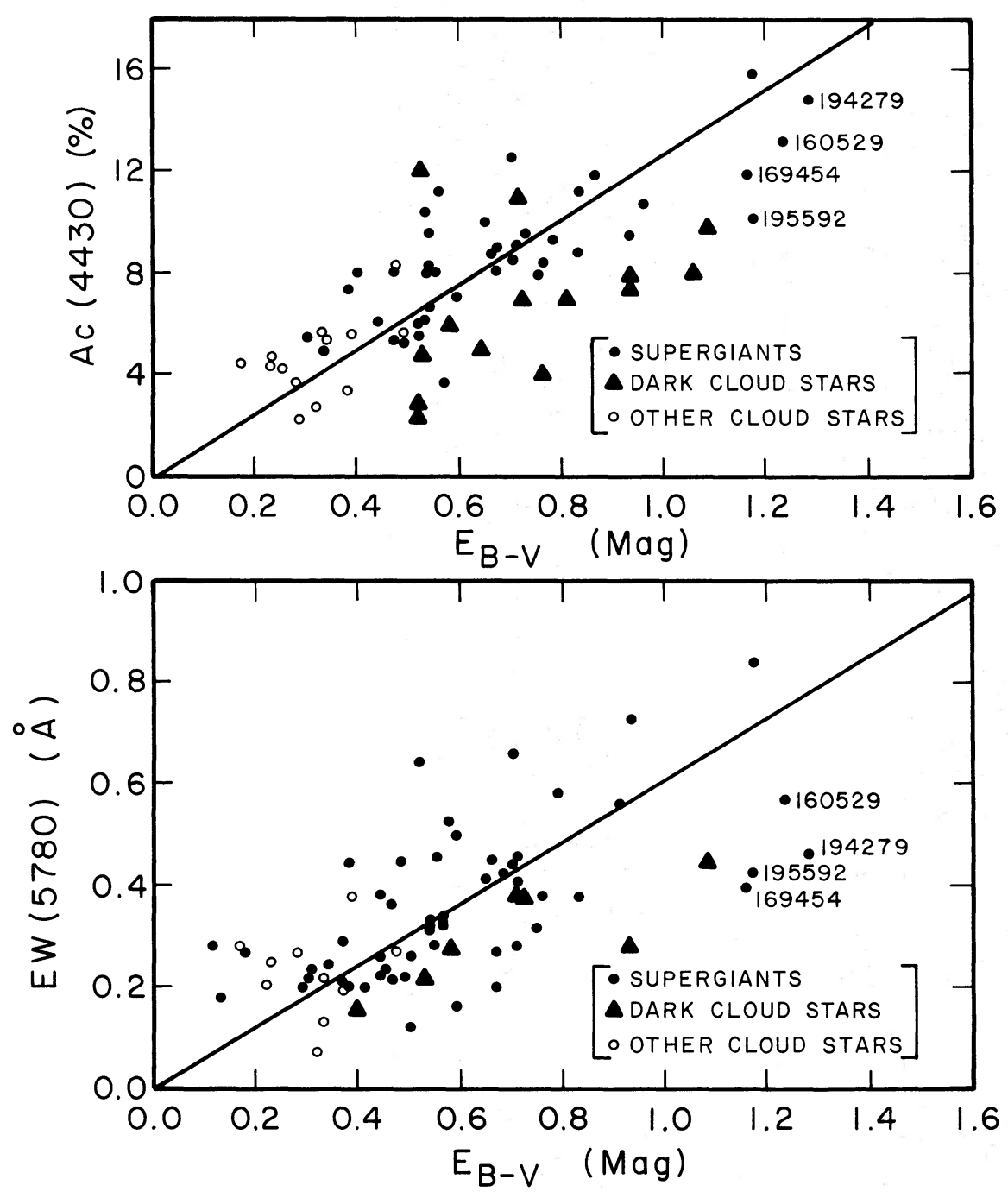

FIG. 1a.-The correlation of the central depth of $\lambda 4430$ with color excess. The solid line indicates the mean correlation based on distant supergiants ( filled circles), forced through the origin. "Dark cloud" and "other cloud" stars (see text) are denoted by triangles and open circles, respectively. $1 b$. The correlation of the equivalent width of $\lambda 5780$ with color excess. The solid line and special symbols have the same meaning as in fig. $1 a$.

made by Duke (1951) or by Walker and Hodge (1966), using the correlation parameters derived by Snow (1973) to convert the values for each star to a common system before averaging them together. In all, nearly 50 stars are represented in the baseline correlation between $\lambda 4430$ and color excess indicated by the filled circles in figure $1 a$. The solid line shows the mean correlation derived by forcing it to pass through the origin. The scatter in the supergiant baseline correlation is generally within $2 \sigma$ of the mean line, with $\sigma=1.5$ percent in the central depth of $\lambda 4430$.

Close to 60 distant supergiants are represented in the baseline correlation between the equivalent width of the $5780 \AA$ feature and $E_{B V}$, which is shown in figure $1 b$. Here also averages were taken in cases where more than one measurement was available for a star. The solid line was derived in a manner similar to the mean $\lambda 4430$ versus $E_{B V}$ correlation indicated in figure $1 a$.

Because $\lambda 5797$ is generally quite weak, and the observational scatter in the data can be large compared with the true band strength, we have not tried to establish correlation of this feature with color excess. We will, instead, examine directly its correlation with $\lambda 5780$ for stars in and out of dark clouds.

\section{COMPARISON OF BAND STRENGTHS IN DARK AND NORMAL CLOUDS}
a) $\lambda \lambda 4430$ and 5780

The "dark cloud" and "other cloud" stars from tables 1 and 2 are denoted by special symbols in figures $1 a$ and $1 b$. In figure $1 a$, which shows the correlation of $\lambda 4430$ strength with color excess, it is 
apparent that the "dark cloud" stars (triangles) tend to fall toward the low side of the scatter, especially for large $E_{B V}$. Many of the triangles are more than $2 \sigma$ away from the mean correlation line. All five of the faint IC 348 stars are deficient in measured $\lambda 4430$ central depth, in spite of their relatively late spectral types. Unresolved blends with stellar lines can cause the central depth of $\lambda 4430$ for A and F stars to be overestimated; any systematic error which may be present in the measured $\lambda 4430$ strengths for these stars is thus most likely to be in the sense that the central depths are too large, and that the stars appear closer to the mean line than they should.

There are two dark-cloud stars, HD 46883 and HD 203938 , which lie well above the mean $\lambda 4430$ versus $E_{B V}$ correlation. It would be of interest to study these stars more thoroughly; first, to confirm the strength of $\lambda 4430$ (we only have one spectrogram of each), and then to measure the extinction and polarization properties as well as other interstellar lines in the foreground clouds to seek evidence of possible peculiarities in the nature of these clouds. The data of Cohen (1973) show that in HD 203938 the interstellar Ca II lines are also enhanced with respect to color excess. Hence in this line of sight, where the gas-to-dust ratio may be peculiar, the $\lambda 4430$ carriers may be distributed more like the gas than the dust. The Ca II lines in HD 46883 are not so obviously discrepant with respect to $E_{B V}$.

No significant general deviation from the mean correlation shown in figure $1 a$ is apparent for the "other cloud" stars (open circles). Because of the criteria used in selecting these stars, they cover a rather narrow range in color excess, and any systematic trend would be difficult to see.

The plot showing the correlation of the strength of the $5780 \AA$ band with color excess (fig. $1 b$ ) indicates that $\lambda 5780$ in dark clouds behaves similarly to $\lambda 4430$. As in the upper plot, the "dark cloud" stars tend to lie below the mean correlation, most notably in the region of large (>0.8 mag) color excesses. It is apparent in the cases of both bands that if a mean correlation of band strength versus color excess were based on the cloud stars alone, the slope would be considerably more shallow than that of the mean correlation based on distant supergiants.

We find in figure $1 b$ that four of the distant supergiants are substantially deficient in the measured equivalent width of the $5780 \AA$ feature. These stars are labeled in figures $1 a$ and $1 b$ with their HD numbers. Two of them, HD 160529 and HD 169454, have extremely high-velocity interstellar-line components which might not derive from galactic rotation (Wallerstein 1970). Possibly the interstellar medium in at least these two lines of sight is influenced by unusual physical conditions. Nothing unusual shows up in the interstellar-line velocities given by Adams (1949) for HD 194279 and HD 195592. It was found by Stoeckly and Dressler (1964) that $\lambda 4430$ tends to be weak in stars with high negative-velocity interstellar-line components. Perhaps the lines of sight to the four supergiants found here to be substantially weak in $\lambda 5780$ strength (and somewhat weak in $\lambda 4430$; see fig. 1a) are subject to conditions similar to those which influence the high-velocity cloud stars of Stoeckly and Dressler. Further study of these lines of sight would be of interest.

Our finding that stars lying behind dense clouds tend to have weak diffuse-band absorption appears not to be consistent with the recent results of Bromage and Nandy (1973), who reported that in the VI Cygni O-B association (Cygnus OB2), where very large color excesses are found, the diffuse bands correlate normally with $E_{B V}$. However, there may be substantial differences between the nature of the extinction in the VI Cygni stars and the "dark cloud" stars in the present study. Perhaps most significant is the fact that both the $\rho$ Ophiuchus and IC 348 cloud complexes, where most of our stars lie, are well out of the galactic plane, and there can be little doubt that the reddening is primarily from the dense clouds in which the observed stars are embedded. Cygnus OB2, on the other hand, is in the galactic plane, and the stars are nearly $2 \mathrm{kpc}$ away (Walborn 1973), so that a very substantial portion of their extinction may be due to the long line of sight through foreground material. In any case, polarimetry data indicate that the grains in the lines of sight to the VI Cygni OB stars do not differ significantly in size from typical interstellar grains (Coyne, Gehrels, and Serkowski 1974), whereas in the $\rho$ Oph cloud, there is evidence that the mean particle size increases with increasing optical depth (CSS). It may be that the $\rho$ Oph and IC 348 cloud complexes are in earlier evolutionary stages than the VI Cygni association, since most of the stars in the former are very young objects detectable only in the infrared (Grasdalen, Strom, and Strom 1973). In any case, the nature of the extinction in the VI Cygni stars shows that they are not embedded in the same kind of dense cloud as are most of the "dark cloud" stars in the present study. Therefore the findings of Bromage and Nandy probably do not contradict ours. Bromage and Nandy do not include $\lambda 4430$ in their study.

\section{b) Correlations among the Diffuse Bands}

In figure $2 a$ is shown the correlation of the equivalent width of $\lambda 5780$ with central absorption of $\lambda 4430$, for distant supergiants ( filled circles), "dark cloud" stars (triangles), and "other cloud" stars (open circles). Although there is a wide variation in the ratio of the strengths of the two bands, as evidenced by the large scatter, there is no evidence that the correlation of $\lambda 5780$ with $\lambda 4430$ in dark clouds is significantly different than in the lines of sight to distant supergiants and "other cloud" stars.

There are two supergiants, HD 13476 and HD 15497 , for which the $\lambda 5780$ band is exceptionally strong with respect to $\lambda 4430$. The data on the $5780 \AA$ feature in both cases come from Merrill et al. (1937), and were given weights of " $A$ " and " $B$," respectively. Hence it is unlikely that the equivalent width of $\lambda 5780$ in these stars is seriously enough in error for the abnormal ratio 


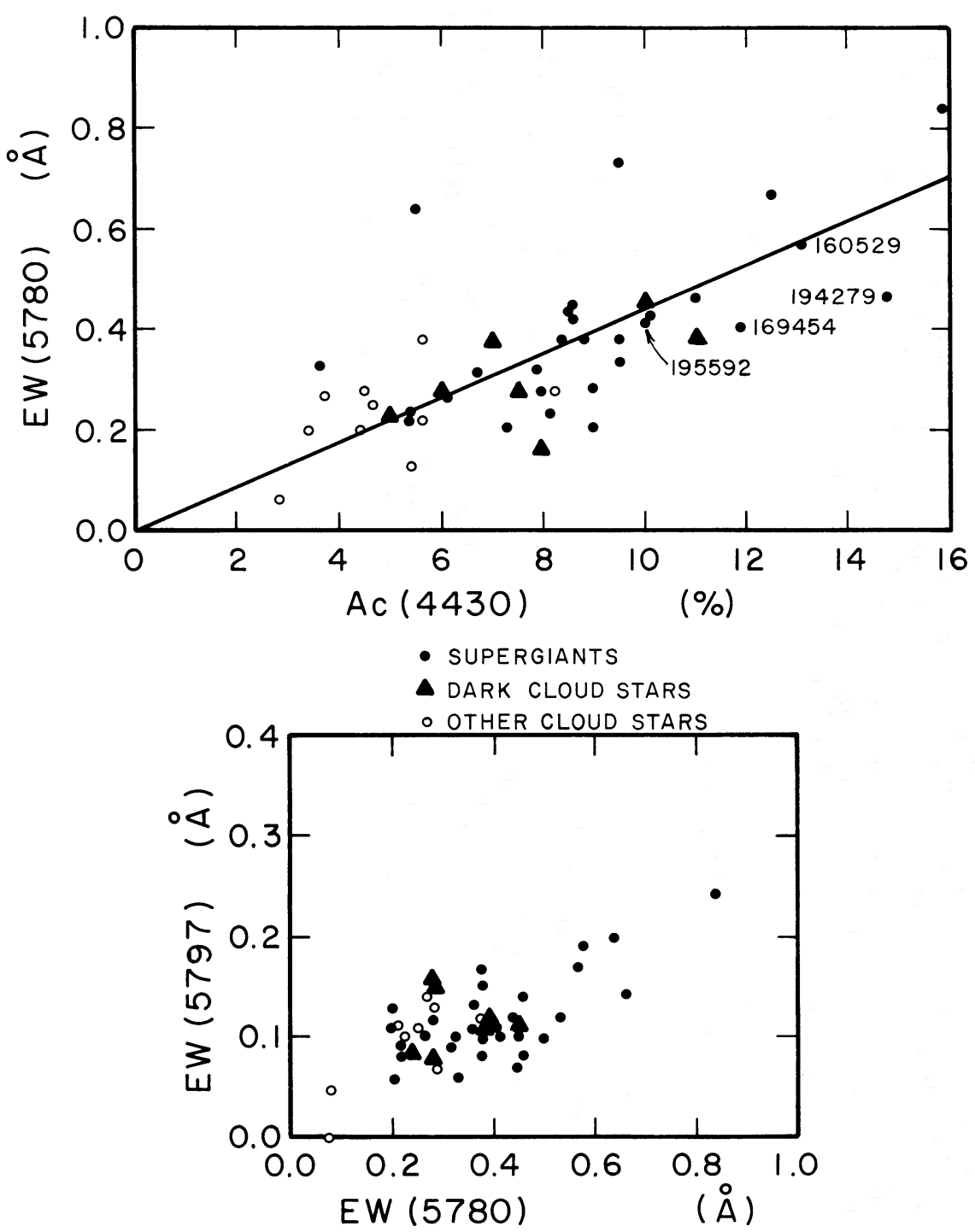

FIG. 2a.-The correlation of $\lambda 5780$ with $\lambda 4430$. Supergiants, "dark cloud" stars, and "other cloud" stars are denoted by filled circles, triangles, and open circles, respectively. The correlation based on distant supergiants, forced through the origin, is indicated by the solid line. $2 b$. The correlation of $\lambda 5797$ with $\lambda 5780$. The symbols are the same as in fig. $2 a$.

of $\lambda 5780$ strength to $\lambda 4430$ central depth to be entirely an observational effect. Both stars have $\lambda 4430$ strengths which place them within $2 \sigma$ of the mean $\lambda 4430$ versus $E_{B V}$ correlation, while the $\lambda 5750$ strengths in both cases place them well above the mean $\lambda 5780$ versus $E_{B V}$ correlation. Similar behavior is noted for VI Cygni No. 10 (Bromage and Nandy 1973). Cases such as these may be taken as evidence that the processes which produce the diffuse bands at 4430 and $5780 \AA$ are not the same. Other examples of discrepant diffuse-band behavior have been pointed out in the literature (e.g., York 1971). Further comparison of the lines of sight to pairs of stars whose ratios of diffuse band strengths differ markedly could be a fruitful area for future research. Whether these cases of peculiar relative band strengths imply the existence of chemically distinct carriers or different physical states in a common carrier is not clear.

The diffuse band at $5797 \AA$ is a difficult one with which to work observationally, because it is generally quite weak. Due to its proximity to $\lambda 5780$ and the generally good correlation with it (e.g., Wu 1972), these two features are often ascribed to a common source. We find no reason to contradict this, even though there is considerable scatter in our $\lambda 5797$ versus $\lambda 5780$ correlation (fig. $2 b$ ). It is not unreasonable to assume that the scatter is dominated by observational effects. It is not possible on the basis of our data to find any obvious systematic strengthening or weakening of $\lambda 5797$ with respect to $\lambda 5780$ in dense clouds. 


\section{THE $\rho$ OPHIUCHI CLOUD COMPLEX}

\section{a) Introduction}

Whether the apparent deficiency of diffuse band strengths in dense clouds is a general characteristic of such clouds or not, it is enlightening to see how the band strengths vary within a well-studied cloud region, where the variation of some of the physical parameters is roughly understood. The $\rho$ Ophiuchi cloud complex, whose extinction and polarization properties have been studied in detail by CSS, is the best case for such a study.

CSS found evidence that the mean grain size within the cloud increases with optical depth. This was based on the fact that the ratio of total to selective absorption and the wavelength of maximum polarization both increases with extinction. Furthermore, data obtained by the Wisconsin experiment on board OAO-2 were cited, which show a decrease in the slope of the ultraviolet extinction curve with increasing optical depth in the cloud, indicating a probable deficiency of small grains in the dense portions of the cloud.

It is argued by CSS that the density in the $\rho$ Oph cloud increases with optical depth, implying that the number of grains in the line of sight, as well as the mean grain size, increases with the optical depth. Hence we will assume that $E_{V K}$, the total extinction, is crudely proportional to the column density of grains, to the extent that an increase in the total extinction implies an increase in the number of particles and vice versa. To make a more quantitative statement would require assumptions about the relative sizes of the grains in different lines of sight, and is not necessary for our purposes.

Cohen (1973) has studied atomic and molecular interstellar lines for some of the stars in the $\rho$ Oph cloud, and her data generally support the conclusion of CSS that the grains in the dense portions of the cloud have grown by depletion of interstellar atomic species. Dividing Cohen's $\mathrm{Ca}$ II and $\mathrm{Na}$ I column densities for $\rho$ Oph cloud stars by the total extinction $E_{V K}$ (from CSS), we find that the ratio $(\log N) / E_{V K}$ decreases systematically with depth in the cloud, confirming that the depletion of these two species is greatest in the most optically thick parts of the cloud.

Based on the work of CSS and of Cohen, one might
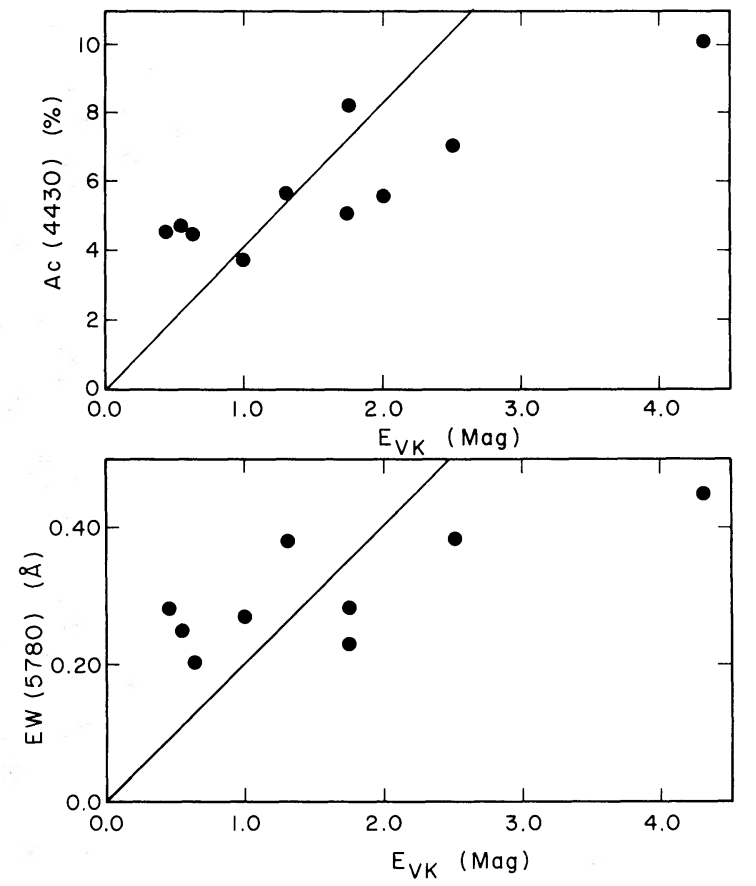

FIG. 3a.-The variation of $\lambda 4430$ with total extinction for stars in the $\rho$ Oph cloud (table 3 ). The solid line is the mean correlation based on distant supergiants, derived by assuming a ratio of total-to-selective absorption of 3 . $3 b$. The correlation of $\lambda 5780$ with total extinction for the $\rho$ Oph cloud. The solid line has the same meaning as in fig. $3 a$. The error bars indicate the observational uncertainty in the vertical positions of the points. The lengths of the error bars were determined by allowing $\pm 2 \sigma$ variations in the measured band strengths. Values of $\bar{\sigma}$ for $\lambda \lambda 4430$ and 5780 are given in the text.

expect to find a systematic variation within the $\rho$ Oph cloud of any properties of the diffuse bands which depend on either the mean grain size or the abundance of some species which itself varies with cloud density.

In table 3 are listed the data on 10 of the $\rho$ Oph cloud stars studied by CSS. Following the star identifications in columns (1) and (2) are the relevant data from CSS, including $E_{V K} / E_{B V}$, the ratio of total to selective absorption (assuming that $E_{V K} \simeq A_{V}$, the total visual extinction); and $\lambda_{\max }$, the wavelength of

TABLE 3

The $\rho$ Ophiuchi Cloud Stars

\begin{tabular}{|c|c|c|c|c|c|c|c|}
\hline Star & $V$ & $E_{B V}$ & $E_{V K}$ & $E_{V K} / E_{B V}$ & $\lambda_{\max }(\AA)$ & $A_{c}(\lambda 4430) / E_{V K}$ & $E W(\lambda 5780) / E_{V K}$ \\
\hline 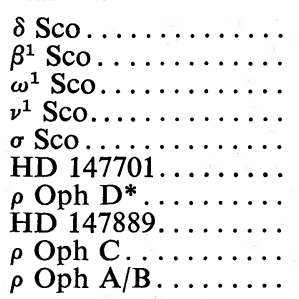 & $\begin{array}{l}2.32 \\
2.61 \\
3.97 \\
4.01 \\
2.93 \\
8.35 \\
6.74 \\
7.86 \\
7.27 \\
4.59\end{array}$ & $\begin{array}{l}0.17 \\
0.23 \\
0.22 \\
0.28 \\
0.39 \\
0.73 \\
0.48 \\
1.09 \\
0.48 \\
0.48\end{array}$ & $\begin{array}{l}0.46 \\
0.56 \\
0.64 \\
1.00 \\
1.32 \\
2.52 \\
1.73 \\
4.30 \\
2.01 \\
1.74\end{array}$ & $\begin{array}{l}2.71 \\
2.43 \\
2.91 \\
3.57 \\
3.39 \\
3.45 \\
3.60 \\
3.94 \\
4.19 \\
3.62\end{array}$ & $\begin{array}{l}6100 \\
5950 \\
7100 \\
5550 \\
7100 \\
8100 \\
6700 \\
6900\end{array}$ & $\begin{array}{l}9.8 \\
8.3 \\
6.9 \\
3.7 \\
4.2 \\
2.8 \\
2.9 \\
2.3 \\
2.7 \\
4.7\end{array}$ & $\begin{array}{l}0.61 \\
0.45 \\
0.31 \\
0.27 \\
0.29 \\
0.15 \\
0.13 \\
0.10 \\
0.16\end{array}$ \\
\hline
\end{tabular}

* $\rho$ Oph D $=$ HD 147888. 
maximum polarization. In the final two columns are shown the ratios $A_{c}(\lambda 4430) / E_{V K}$ and $E W(\lambda 5780) / E_{V K}$, which are taken as measures of the diffuse bandproducing efficiency of the grains in each line of sight.

\section{b) Variation of Band Strengths within the Cloud}

In figures $3 a$ and $3 b$ are shown the correlations of $\lambda \lambda 4430$ and 5780 with total extinction in the $\rho$ Oph complex. There is a general increase of band strengths with increasing optical depth, but the rate of increase at large depths is apparently much slower than in a typical line of sight through many "normal" interstellar clouds, as indicated by the solid lines. These mean correlations of band strengths with total extinction were derived from the mean correlations shown in figure 1, with the assumption that $E_{V K}=3 E_{B V}, 3$ being the commonly assumed ratio of total-to-selective absorption in typical clouds. The fact that the points representing stars at large depths in the cloud tend to fall below the solid line indicates that the $\rho$ Oph cloud in particular follows the general trend indicated by
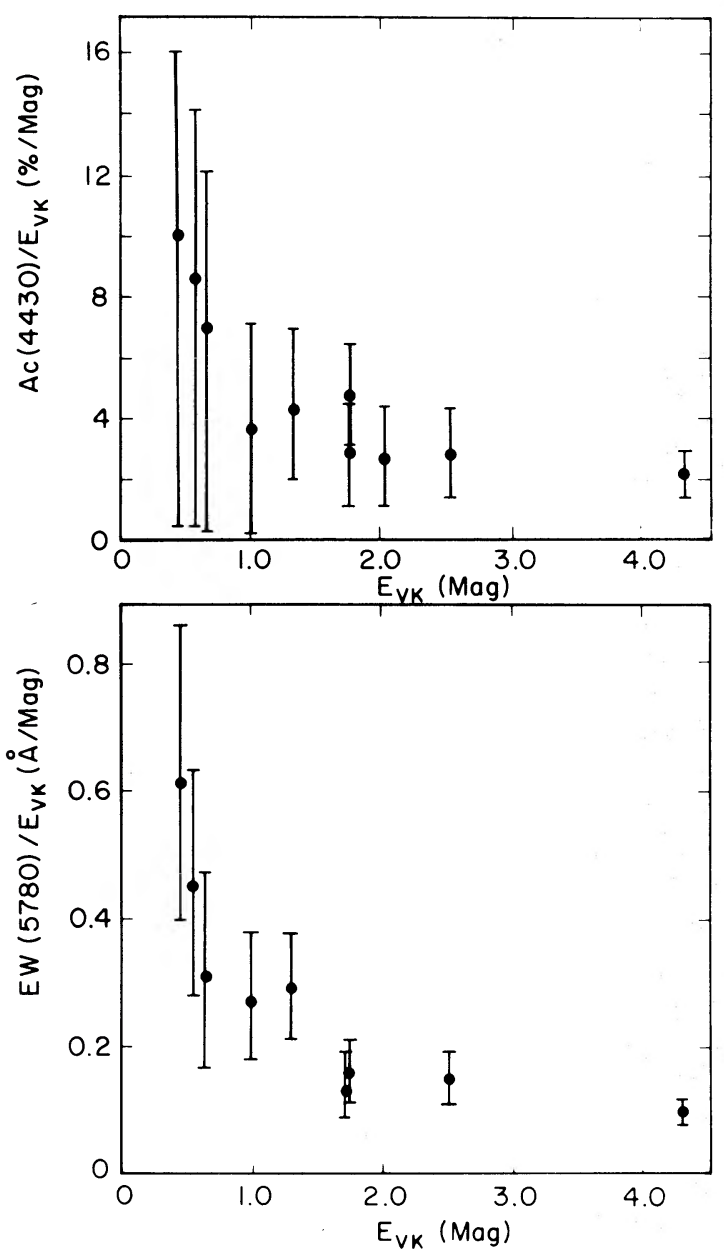

FIG. 4a.-The variation of $\lambda 4430$ strength per grain with total extinction in the $\rho$ Oph cloud. $4 b$. The same plot, with $\lambda 5780$ in place of $\lambda 4430$.
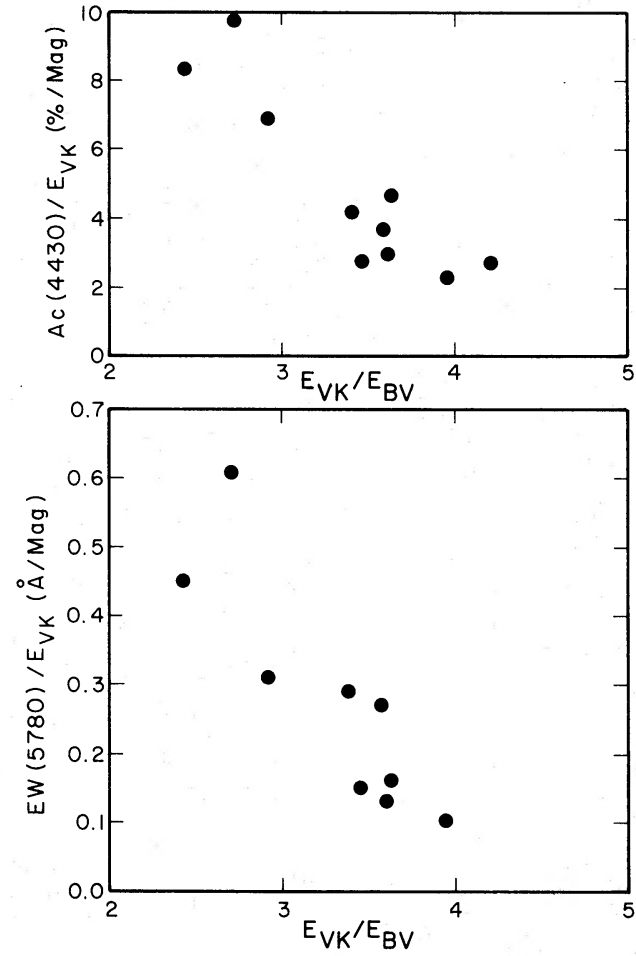

FIG. 5a.-The variation of $\lambda 4430$ strength per grain with the ratio of total to selective absorption for stars in the $\rho$ Oph cloud. $5 b$. The same plot, for $\lambda 5780$.

figure 1. It is unfortunate, however, that data are not available for more deeply embedded $\left(E_{V K}>1.5 \mathrm{mag}\right)$ stars in the $\rho$ Oph complex, because the effect shown in figure 3 depends heavily on one star, HD 147889.

The slow increase of band strengths with extinction in dense portions of the $\rho$ Oph cloud may be an indication that the most efficient band formation is taking place in the outer regions, where the densities are lower. This is borne out by an examination of the way in which the ratios $A_{c}(\lambda 4430) / E_{V K}$ and $E W(\lambda 5780) / E_{V K}$ vary with extinction. These ratios, which roughly measure the band strength per grain if we assume that the total extinction is related to the number of grains in the line of sight, can be taken as indicators of the band-producing efficiency in the various lines of sight through the cloud. It is interesting to see how this efficiency varies with optical depth and especially with mean grain size.

We show in figures $4 a$ and $4 b$ the dependence of $A_{c}(\lambda 4430) / E_{V K}$ and $E W(\lambda 5780) / E_{V K}$ on total extinction $E_{V K}$. These plots imply that the band strength per grain decreases with optical depth in the cloud.

It is perhaps most indicative to plot directly the band-forming efficiency against indicators of grain size. In figures $5 a$ and $5 b$ are shown the variations of $A_{c}(\lambda 4430) / E_{V K}$ and $E W(\lambda 5780) / E_{V K}$ with ratio $E_{V K} / E_{B V}$, which is in some sense proportional to mean grain size. In figures $6 a$ and $6 b$ are shown the dependence of $A_{c}(\lambda 4430) / E_{V K}$ and $E W(\lambda 5780) / E_{V K}$ on $\lambda_{\max }$, which is 


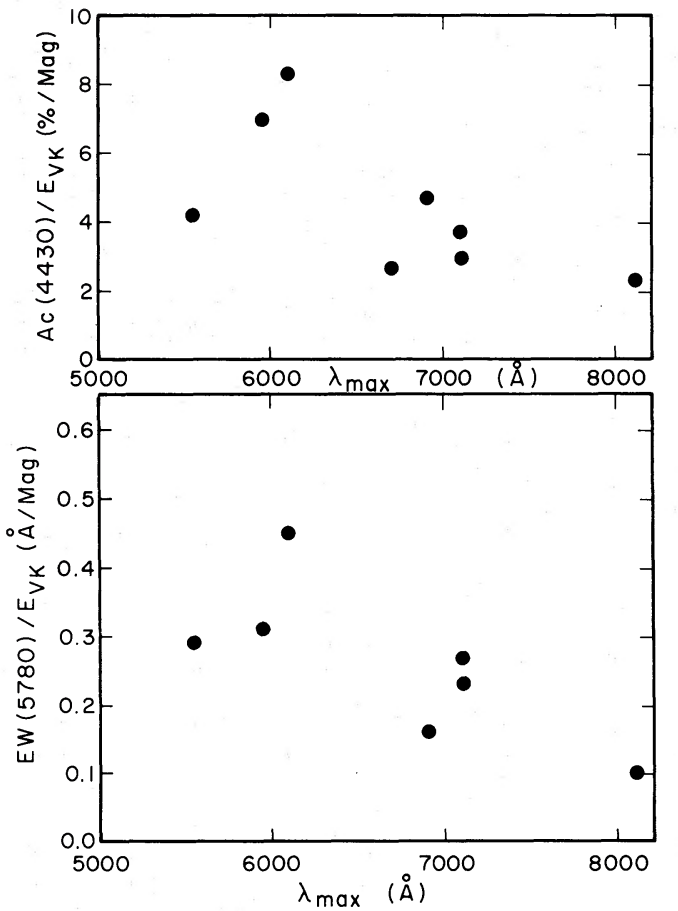

FIG. $6 a$. - The variation of $\lambda 4430$ strength per grain with the wavelength of maximum interstellar polarization for the $\rho$ Oph stars. $6 b$. The same plot, using $\lambda 5780$ strength in place of $\lambda 4430$. This figure, along with fig. 5 , shows that the bandproducing efficiency of the grains decreases with increasing grain size.

another indicator of grain size. The strengths of both $\lambda 4430$ and $\lambda 5780$ per grain are seen to decrease with increasing grain size. It is very clear that the bandproducing capability of the grains does not increase in proportion to the size of the grains.

Data on the diffuse band at $6284 \AA$ are available in Merrill et al. (1937) for five of the $\rho$ Oph cloud stars, and for these stars the ratio $E W(\lambda 6284) / E_{V K}$ varies with $E_{V K} / E_{B V}$ in a manner similar to that shown in figure 5 for the 4430 and $5780 \AA$ features. This is consistent with Murdin's (1972) find that $\lambda 6284$ may be perfectly correlated with $\lambda 4430$.

Plots of the ratios $E W(\lambda 5780) / A_{c}(\lambda 4430)$ and $E W(\lambda 5797) / E W(\lambda 5780)$ against $E_{V K} / E_{B V}$ show no significant trend. It does not appear that the strength of any one of the bands is a stronger or weaker function of grain size than that of any of the other bands. This is consistent with the lack of systematic variations in the ratios of band strengths in and out of dark clouds, which is indicated in figure 2 .

\section{DISCUSSION}

We can make some general comments on the possible origins of the diffuse bands, based on what we have learned about their behavior in dense clouds.

The most common assumption concerning the origin of the bands is that they are formed by some process in solid grains. This assumption is based primarily on two observational findings: (1) the general correla- tion of band strengths with color excess, and (2) difficulties with hypotheses of band formation in molecules, including primarily the lack of observable fine structure (Herbig 1966; Wu 1972) and the improbability of sustaining a great enough abundance of the carrier molecule in space (Wilson 1964). For a review of many of the models attributing the bands to solid grains, see Aannestad and Purcell (1973). It is pointed out in that paper that the lack of variability of band profile and central wavelength exercises a constraint on the nature of the grains, because such variations would be expected to occur with changes in grain size or concentration of absorbers. Since it was already established that the mean grain size probably increases systematically with increasing depth in the $\rho$ Oph cloud (CSS), and our findings imply that the volume density of diffuse-band absorbers per grain decreases systematically with increasing depth, it would be very important to carry out a careful study of the diffuseband profiles throughout the $\rho$ Oph cloud. If no variations of central wavelength or profile are found, then one must seriously consider sources of the diffuse bands other than the grains which produce the optical extinction. The very important question of whether or not polarization structure exists within the bands remains unresolved, opposing results having been reported recently by Martin and Angel (1974) and by Wolstencroft and Kemp (1974). These results are suggestive that $\lambda \lambda 4430$ and 5780 absorption is not always accompanied by as great a variation of polarization with wavelength as would be expected if the bands are formed in the same grains which produce the general interstellar polarization.

From the present work we can conclude that the bands very probably are not formed in the mantles of solid grains. Such mantles are apparently most strongly in evidence inside dense clouds, where we have shown that the band-forming efficiency of the grains is low. Suggestions specifically attributing the formation of diffuse bands to grain mantles have been studied in some detail by Duley and Graham (1969, 1971), Graham and Duley (1971) and McCullough et al. (1972).

On the other hand, it may still be consistent to assume that the bands are formed in small, mantleless grains, and that the thick coatings accreted by grains within dense clouds smother the optical properties of the cores. The suggestion of Stoeckly and Dressler (1964) that $\lambda 4430$ is produced by small grains which tend to be destroyed in the presence of strong heating cannot be ruled out if grains of this type can form the cores of the larger grains in dense clouds. Grain destruction by radiation from the hot stars embedded in the clouds probably cannot account for the deficiency of the diffuse bands (Wampler 1966).

We cannot, on the basis of our present findings, rule out the somewhat less popular possibility that the diffuse bands are produced in free molecules. The lack of fine structure within the bands is often taken as a definitive criticism of the kind of band source, but the limits placed on the possibility of structure being present are not severe enough to rule it out totally 
(Martin and Angel 1974). Furthermore, molecular predissociation transitions would not be expected to show fine structure, and hence could account for the observed profiles (Herzberg 1967). There are other possible kinds of molecular transitions which would not show fine structure and would also not destroy the molecule (Smith 1974).

We can conclude that, if some molecule is responsible for diffuse-band formation, it is not $\mathrm{H}_{2}$ or any other species which would be most abundant in the densest parts of the interstellar clouds. Molecular hydrogen in matrix was suggested by Herbig (1963) as a possible source of $\lambda 4430$, but energy considerations have already made this seem to be an unlikely origin of the band, because it is difficult to get $\mathrm{H}_{2}$ into the required excited state. It has been found from data obtained by the Copernicus satellite that no significant fraction of the $\mathrm{H}_{2}$ in space is in any but the lowest two rotational levels of the ground electronic and vibrational states (Spitzer, Drake et al. 1973; Spitzer and Cochran 1974; Spitzer, Cochran, and Hirshfeld 1974). These data are consistent with arguments based on selfshielding of $\mathrm{H}_{2}$ (Hollenbach, Werner, and Salpeter 1971) that molecular hydrogen is dominant in the interiors of interstellar clouds; hence we would expect the diffuse bands to be formed most efficiently in the same regions, if their carriers were distributed like $\mathrm{H}_{2}$. We have shown that this is not the case.

There are molecular species for which one might expect the greatest abundances in the outer portions of interstellar clouds, however. These would include many hydrogen-bearing molecules, since $\mathrm{H}_{2}$ probably dominates so strongly in cloud interiors that little hydrogen would be available to form other molecular species. Also included would be molecules whose formation requires abundant ultraviolet flux. An examination of Cohen's (1973) data shows that interstellar $\mathrm{CH}$ and $\mathrm{CH}^{+}$display this kind of behavior; for example, the ratio $\left(\log N_{\mathrm{CH}}\right) / E_{V K}$ decreases with optical depth in the $\rho$ Oph cloud in a manner similar to the ratios $A_{c}(\lambda 4430) / E_{V K}$ and $E W(\lambda 5780) / E_{V K}$. It would not be inconsistent with our findings on the behavior of the diffuse bands in dense clouds to assume that they originate in some molecule (or molecules) which are distributed like $\mathrm{CH}$ and $\mathrm{CH}^{+}$. That many as yet unidentified molecules may be quite abundantly present in space is suggested by the many strong unidentified interstellar lines found in the ultraviolet spectra of reddened stars obtained by the Copernicus satellite (Morton 1974).

If the bands are produced by some molecules which are most abundant in the outer portions of interstellar clouds, then the general correlation of band strength with extinction is a reflection of a fairly constant ratio of the abundances of these molecules to the number of grains in the line of sight. In this view, the apparently normal correlation between band strengths and extinction in the VI Cygni association would be an indication that the abundances of the band-producing molecules are normal in that region, rather than being low as in the dense clouds included in the present study. Quite possibly it is easier to understand variations in molecular abundances in different regions of space than it is to understand variations in the nature of interstellar grains. The substantial fluctuations noted for a few cases in the relative strengths of $\lambda 5780$ and 4430 might imply that at least two distinct molecular species may be responsible for the observed bands.

\section{SUMMARY}

We can base several conclusions on our findings in this study, which must be taken into account in considerations of possible band sources.

Our primary findings are: (1) The diffuse interstellar bands tend to be weak with respect to extinction in the dense clouds considered. There is no evidence of systematic selective weakening of any band or bands over any others. (2) The diffuse band strength per grain (as indicated by the ratio $S / E_{V K}$, where $S$ is the band strength in appropriate units) does not increase with increasing optical depth and with increasing grain size in the $\rho$ Oph cloud complex, and in fact probably decreases. This decrease with optical depth and grain size is strongly indicative that neither the large grains nor the molecular species which are dominant in the interiors, or such clouds are the major source of diffuse-band formation. (3) Our suggestion that the most efficient diffuse-band formation takes place in the outer portions of interstellar clouds can be consistent with a band origin either in small grains which are not present in cloud interiors, presumably because they have been coated with thick mantles in those regions; or in molecules which are most abundant in the outer portions of interstellar clouds. Our data are not consistent with suggestions that the diffuse bands are formed on the surfaces of grains which bear mantles.

The authors wish to acknowledge the kind assistance of Dr. S. Strom, who provided diffuse-band data on five stars, and Dr. G. Wallerstein, who provided plates of some of the $\rho$ Oph cloud stars. One of us (T. P. S.) carried out a portion of this study while he was a Predoctoral Research Associate at the University of Washington, where his research was supported in part by the Graduate School Research Fund of the University of Washington, and in part by National Science Foundation grant GP-28881. The work done at Princeton was supported by National Aeronautics and Space Administration contract NAS5-1810.

\section{REFERENCES}

Aannestad, P. A., and Purcell, E. M. 1973, Ann. Rev. Astr. and $A p ., 11,309$.

Adams, W. S. 1949, Ap. J., 109, 354.
Bromage, G. E., and Nandy, K. 1973, Astr. and Ap., 26, 17. Carrasco, L., Strom, S. E., and Strom, K. M. 1973, Ap. J., 182, 95 (CSS). 
Cohen, J. G. 1973, Ap. J., 186, 149.

Coyne, G. V., Gehrels, T., and Serkowski, K. 1974, A.J., 79, 581.

Deeming, T. J., and Walker, G. A. H. 1967, Zs. f. Ap., 66, 175.

Duke, D. 1951, Ap. J., 113, 100.

Duley, W. W., and Graham, W. R. M. 1969, Nature, 224, 785. 1971, Astr. Nach., 293, 33.

Field, G. B. 1974, Ap. J., 187, 453.

Field, G. B., Goldsmith, D. W., and Habing, H. J. 1969, Ap.J. (Letters), 155, L149.

Fredrick, L. W. 1956, A.J., 61, 437.

Graham, W. R. M., and Duley, W. W. 1971, Nature Phys. Sci., 232, 43.

Grasdalen, G. L., Strom, K. M., and Strom, S. E. 1973, Ap. J. (Letters), 184, L53.

Harris, D. L., Morgan, W. W., and Roman, N. G. 1954, Ap. J., 119, 622.

Herbig, G. H. 1963, Ap. J., 137, 200.

—_. 1966, Zs. f. Ap., 64, 512.

Herzberg, G. 1967, IAU Symposium No. 31, Radio Astronomy and the Galactic System, ed. H. van Woerden (New York: Academic Press), p. 91.

Hollenbach, D., Werner, M. W., and Salpeter, E. E. 1971, Ap. J., 163, 165.

Krzemiński, W. 1959, Acta Astr., 9, 23.

Lynds, B. T. 1962, Ap. J. Suppl., 7, 1.

Martin, P. G., and Angel, J. R. P. 1974, Ap. J., 188, 517.

McCullough, J. D., Floyd, G. R., Prince, R. H., and Duley, W. W. 1972, IAU Symposium No. 52, Interstellar Dust and Related Topics, ed. J. M. Greenberg and H. C. van de Hulst (Boston: Reidel), p. 327.
Merrill, P. W. 1934, Pub. A.S.P., 46, 206. 1936, Ap.J., 83, 126 .

Merrill, P. W., Sanford, R. F., Wilson, O. C., and Burwell, C. G. 1937, Ap.J., 86, 274.

Merrill, P. W., and Wilson, O. C. 1938, Ap. J., 87, 9.

Mészáros, P. 1972, Ap. J., 177, 79.

Morton, D. C. 1974, private communication.

Murdin, P. 1972, M.N.R.A.S., 157, 461.

Smith, W. H. 1974, private communication.

Snow, T. P. 1973, A.J., 78, 913.

Spitzer, L., and Cochran, W. D. 1974, Ap. J. (Letters), 186, L13.

Spitzer, L., Cochran, W. D., and Hirshfeld, A. 1974, Ap. J., preprint.

Spitzer, L., Drake, J. F., Jenkins, E. B., Morton, D. C., Rogerson, J. B., and York, D. G. 1973, Ap. J. (Letters), 181, L110.

Stoeckley, R., and Dressler, K. 1964, Ap. J., 139, 240.

Strom, S. E. 1974, Pub. A.S.P., in press.

Walborn, N. R. 1973, Ap. J. (Letters), 180, L35.

Walker, G. A. H. 1963, M.N.R.A.S., 125, 141.

Walker, G. A. H., and Hodge, S. M. 1966, Pub. Dom. Ap. Obs., 12, 401.

Wallerstein, G. 1970, Pub. A.S.P., 82, 8.

Wallerstein, G., and Goldsmith, D. 1974, Ap. J., 187, 237.

Wampler, E. J. 1966, Ap. J., 144, 921.

Wilson, R. 1964, Pub. Roy. Obs. Edinburgh, 4, 67.

Wolstencroft, R. D., and Kemp, J. C. 1974, preprint.

Wu, C.-C. $1972, A p . J ., 178,681$.

York, D. G. 1971, Ap. J., 166, 65.

Judith G. Cohen: Kitt Peak National Observatory, P.O. No. 26730, Tucson, AZ 85726

Theodore P. Snow: Dept. of Astrophysical Sciences, Peyton Hall, Princeton University, Princeton, NJ 08540 